\title{
Efficacy of Acupuncture On Cognitive Function In Poststroke Depression: Study Protocol For A Randomized, Placebo-Controlled Trial
}

Ling Chen ( $\nabla$ chenling@gzucm.edu.cn )

Guangzhou University of Chinese Medicine

Yi Chen

Guangzhou University of Chinese Medicine

Lihua Wu

Guangzhou University of Chinese Medicine

Wen Fu

Guangzhou University of Chinese Medicine

\section{Luanmian Wu}

Guangzhou University of Chinese Medicine

Wenbin Fu

Guangzhou University of Chinese Medicine

\section{Study protocol}

Keywords: Poststroke depression, Acupuncture, Efficacy, Cognitive function, Randomized controlled trial, Clinical trial

Posted Date: June 28th, 2021

DOl: https://doi.org/10.21203/rs.3.rs-76109/v1

License: (c) (i) This work is licensed under a Creative Commons Attribution 4.0 International License. Read Full License 


\section{Abstract}

Introduction: Poststroke depression (PSD) is the most common mental complication after stroke and has a serious impact on functional outcomes and quality of life. Antidepressants are the first-line treatment for PSD, but many reported side effects remain. Clinical research has shown that acupuncture has a positive effect on PSD. This trial aims to study the efficacy and safety of acupuncture for PSD and to explore its effect on cognitive function. It is hypothesized that acupuncture treatment improves depressive symptom, cognitive behavior and negative emotion processing bias in PSD.

Methods: In this randomized, placebo-controlled, single-blinded trial, fifty-six people with PSD will be randomly allocated into the intervention $(n=28)$ or control $(n=28)$ groups. The intervention group will receive acupuncture treatment, and the control group will receive sham acupuncture treatment, in 20 sessions over 4 weeks. The primary outcome is the change from baseline in the Hamilton Depression Scale-17 (HAMD-17) scores at week 4. Secondary outcomes include the Wisconsin Card Sorting Test (WCST) and latency and amplitude of P1, N170 and P3 of the event-related potentials (ERPs) components to assess the changes in cognitive function and electroencephalography. Outcomes are assessed at baseline and post intervention.

Discussion: Acupuncture therapy could become an alternative treatment for PSD, and it is expected that this trial will provide reliable clinical evidence for the future use of acupuncture for the treatment of PSD.

Trial registration: Chinese Clinical Trial Registry: ChiCTR1900026948. Registered on 27 October 2019.

\section{Introduction}

Stroke is one of the most devastating diseases affecting the aging population in the world and is the main cause of death and disability [1]. Poststroke depression (PSD) is the most frequent emotional disorders resulting from stroke and characterized by hopelessness and loss of interest and pleasure beyond stroke symptoms. Approximately $33 \%$ of stroke survivors suffer from depression [2], which is 4 to 10 times the incidence observed in the general population [3]. PSD not only increases the risk of stroke recurrence [4] but also affects functional outcomes and quality of life [5]. Moreover, the mortality rate in stroke survivors with PSD is 3 to 4 times higher than that in stroke survivors without depression [6]. Although stroke is the direct cause of PSD, the underlying mechanisms of PSD remain unclear. At present, the diagnosis of PSD is mainly based on clinical manifestations combined with scale assessments, as there is a lack of physiological indicators. As approximately one-third of stroke patients experience aphasia [7], 70\% of stroke survivors have cognitive impairment [8]. It is difficult to know whether emotion and interest have changed, which poses a great challenge in the treatment of PSD.

Pharmacotherapy is the first-line treatment of PSD. However, antidepressants are ineffective in approximately $40-60 \%$ of patients [9], and $30 \%$ of patients develop refractory depression due to ineffective treatment with multiple antidepressants [9]. In addition, several antidepressants increased the 
risk of cardiovascular disease and stroke [10], resulting in a low treatment compliance rate. Therefore, there is an urgent need for therapy that is effective, safe and easy to promote.

Acupuncture is recommended as a promising nonpharmaceutical therapy for major depressive disorder (MDD). Published literature [11] indicates that acupuncture could improve the symptoms of depression evidenced by reductions in Hamilton Depression Scale (HAMD) scores in individuals with PSD. An fMRI study demonstrated that acupuncture may have an antidepressant effect by regulating emotion-related brain areas such as the amygdala and anterior cingulate cortex [12]. Several meta-analyses and systematic reviews have shown that acupuncture may be superior to antidepressants in alleviating the depressive symptoms of PSD $[11,13]$. However, these trials did not consider placebo or sham acupuncture treatment to exclude the psychological effects of acupuncture.

Studies have shown that depression is associated with cognitive control deficits with regard to emotional information [14]. MDD is characterized by abnormal cognitive processing of negative bias, which is manifested by paying excessive attention to negative emotional information $[15,16]$. The cognitive processing bias of negative emotions penetrates the whole process of depression and is at the core of the development of depressive symptoms. It is very important to understand the effects of acupuncture on cognition as it relates to PSD because understanding the relationship between emotions and cognitive function can help to explain the antidepressant effects. Event-related potentials (ERPs) reflect neural activities in the cognitive process, and because their assessment is noninvasive and has high time resolution (milliseconds), ERP measurements have become the preferred technology for studying neurocognition. According to a recent study, the recurrence and severity of depression may increase disrupted emotional processing, and the ERP component may be a potential biomarker to predict depression and its recurrence [17].

This randomized, placebo-controlled, single-blinded study was designed to explore the efficacy and safety of acupuncture on PSD and to explore its effect on cognitive function. It is hypothesized that acupuncture treatment will improve symptoms of depression, cognitive function and abnormal negative emotion processing bias in individuals with PSD.

\section{Methods}

\section{Study setting}

This randomized, placebo-controlled, single-blinded trial will recruit 56 stroke survivors with depression. This study will be conducted at the Department of Acupuncture of The Secondary Medical College of Guangzhou University of Traditional Chinese Medicine, China. The study protocol was registered at the Chinese Clinical Trial Registry (ChiCTR1900026948). The study will be performed in line with the Declaration of Helsinki formulated by the World Medical Association. Written informed consent will be obtained from all participants. All routine interventions will be performed by hospital acupuncture staff. Figure 1 shows the CONSORT flowchart of the trial. This protocol was organized based on the Standard 
Protocol Items: Recommendations for Interventional Trials (SPIRIT) Checklist (Additional file 1) [18]. Figure 2 shows the SPIRIT outline for enrollment, interventions, and assessments.

\section{Eligibility criteria}

\section{Inclusion criteria}

The inclusion criteria are as follows: (1) patient meets the criteria for "stroke" and "depression" based on the ICD-10 and are diagnosed with PSD by a psychiatrist; (2) patient is aged between 40 and 75 years old; (3) ischemic stroke was confirmed by computed tomography (CT) or magnetic resonance imaging (MRI); (4) patient shows syndromes of liver stagnation based on traditional Chinese medicine (TCM) diagnostic criteria; (5) patient has a HAMD-17 score $\geq 17$; and (6) the duration of PSD is less than 6 months. For the TCM diagnostic criteria, we refer to "Expert Consensus of Diagnosis and Treatment of Major Depression with Integrated TCM and Western Medicine" [19].

\section{Exclusion criteria}

The exclusion criteria are as follows: (1) massive cerebral infarction; (2) suicidal tendency as evaluated by specialists; (3) serious medical diseases, such as brain injury, epilepsy, malignant tumor, congestive heart failure, respiratory failure, renal failure and atrial fibrillation; (4) previous head trauma history, such as coma, vomiting, amnesia, convulsions or medical emergency after injury; (5) severe cognitive impairment (Mini-Mental State Examination $\leq 21$ [20]); (6) schizophrenia, anxiety or other mental disorders; (7) a DSM-IV history of substance abuse or dependence in the 6 months prior to assessment; and (8) metal implants in the body, such as pacemaker, deep brain stimulator and vascular stent.

\section{Intervention}

The enrolled PSD participants receive basic treatment for stroke: basic pharmacotherapy treatment for ischemic stroke and other diseases (such as hypertension, coronary heart disease and diabetes, etc.), and physical therapy and occupational therapy for motor dysfunction will occur every day over $60 \mathrm{~min}$ (including preparation). The acupuncture group will receive acupuncture treatment, and the control group will receive sham acupuncture treatment. The participating physicians in the trial are acupuncture doctors at the Secondary Medical College of Guangzhou University of Traditional Chinese Medicine. As the acupuncturists are responsible for the acupuncture treatment, they are all highly qualified, have a master's degree in acupuncture and moxibustion, have undergone training in a unified standardized operation plan. Acupuncture treatment will be administered in the supine position, and the temperature of the treatment room is maintained at $26^{\circ} \mathrm{C}$. Participants will receive 20 treatment sessions over a 4-week period at a frequency of 5 times per week.

\section{Acupuncture group}


Participants in the acupuncture group will receive acupuncture at Baihui (GV20), Yingtang (GV29), Hegu (LI4, bilateral), and Taichong (LR3, bilateral). Disposable stainless-steel needles $(0.3 \mathrm{~mm} \times 40 \mathrm{~mm}$; Suzhou Tianxie Acupuncture Instruments Co., Ltd., Suzhou City, China) will be used. After skin disinfection, sterile adhesive pads will be placed on the acupoints. For GV20 and GV29, acupuncture needles will be inserted through the adhesive pads for approximately $15 \mathrm{~mm}$ at an angle of 15 degrees. For bilateral LI4 and LR3, the needles will be inserted vertically to a depth of $20 \mathrm{~mm}$. Following needle insertion, the acupuncturists will twirl the needle handles back and forth to achieve the sensation of achiness, heaviness and numbness (known as de qi) at all acupoints. The needles will be removed after 20 min.

\section{Control group}

Participants in the control group will receive sham acupuncture at the same acupoints. In the sham acupuncture group, placebo needles with a blunt tip $(0.3 \mathrm{~mm} \times 40 \mathrm{~mm}$; Suzhou Tianxie Acupuncture Instruments Co., Ltd., Suzhou City, China) and sterile adhesive pads will be used. The placebo needles are needled through the pads pressed against the skin without penetration. The procedures and other treatment parameters will be the same as those in the acupuncture group, e.g., the needles will be left in place for $20 \mathrm{~min}$, but there will be no acupuncture needle manipulation.

\section{Follow-up}

To evaluate the persistence of the effects, a follow-up assessment will occur 1 month after the end of the 4-week treatment.

\section{Outcome measures}

The endpoints will be evaluated at baseline (before intervention), week 4 (immediately after intervention), and week 8 (1-month follow-up). We include two figures to show the participant timeline more clearly (see Figs. 1 and 2).

\section{Primary outcome}

The primary outcome is the change in the degree of depression as measured by the HAMD-17 [21] at week 4 . The HAMD-17 consists of a total of 13 items with a total score that can range from $0-24$, where 0 defines least depressed and 24 describes most depressed.

\section{Secondary outcomes}

The secondary outcomes include the Wisconsin Card Sorting Test (WCST) and the latency and amplitude of the ERP components P1, N170 and P3 at week 4.

- The WCST [22] is being adopted to assess cognitive function. Participants are required to match response cards to the four stimulus cards along one of the three dimensions (color, form, or number) 
by pressing one of the 1 to 4 number keys on the computer keyboard. The measured variables include categories completed (CC), total errors (TE), perseverative errors (PE), and nonperseverative errors (NRPE).

- ERP

\section{Stimuli.}

Facial pictures are grayscale photographs selected from the native Chinese Facial Affective Picture System [16], and a total of 60 facial pictures are used as target faces (20 happy, 20 neutral and 20 fear faces) with an equal number of males and females. Each picture was assessed for its valence and arousal on a 9-point scale with a large sample of Chinese participants in a previous survey. Each pair of facial stimuli will consist of a neutral picture and an emotional picture. Thus, we will have pairs of emotional-face stimuli consisting of 60 emotional faces, including 20 happy-neutral, 20 sad-neutral, and 20 fear-neutral pairs of facial stimuli.

\section{Procedure.}

The experimental paradigm is a modified dot-probe paradigm. The task consists of 160 trials. A central fixation appears for $300 \mathrm{~ms}$, and then replaced by a pair of emotional-face stimuli with different valences as a cue, which will last for $500 \mathrm{~ms}$. After a short 200-ms interval, the dot probe is randomly presented for $150 \mathrm{~ms}$ as a target in either the left or right position of the fixation cross. The participant is asked to identify the spatial location of the 'dot' and register the response with the forefinger pressing the button ' 1 ' or ' 4 ' on the reaction box as quickly as possible. An automatic interval of $2000 \mathrm{~ms}$ is programmed to receive the participants' response; after a response is made or $2000 \mathrm{~ms}$ (whichever occurs first), a black screen is presented for $600 \mathrm{~ms}$ and followed by the subsequent trial [23].

\section{ERP data}

During the cognitive task, ERP data will be recorded by the NeuroScan ERP system (NeuroScan Company, American), which contains a 64-channel amplifier and a 64-electrode $\mathrm{Ag} / \mathrm{AgCL}$ scalp cap. Each epoch following the emotional-face cue and target will mainly be analyzed by an off-line analysis using MATLAB 2013a (The Mathworks, Natick, USA) as well as its toolbox ERPLAB toolbox (http://www.erpinfo.org/erplab). The latency and average amplitudes of the three ERP components P100, $\mathrm{N} 170$, and P300 will be recorded and have been detected in various stages of attention processing.

\section{Behavioral data}

E-Prime software (Psychology Software Tools Inc.) will be applied to program the experimental procedure, record the accuracy of the responses, and the behavioral reaction times (RT) together with the ERP data.

\section{Safety evaluation}


Adverse events (AEs) during the treatment will be recorded. AEs include the following: hematoma around the site of needling insertion points, local numbness, pain, and dizziness during treatment. If any $A E$ occurs, the doctor will assess and provide corresponding treatment to the patient in accordance with the study protocol, and report the adverse events form in the case report form.

\section{Blinding assessment}

The acupuncturists will ask participants to guess whether they received acupuncture treatment or sham acupuncture after the last treatment in week 4 .

\section{Sample size}

The study uses the HAMD-17 as the primary outcome measure for sample size calculation. The sample size was calculated using the following formula for the comparison of two samples:

$n=\frac{\left(Z_{1-\frac{a}{2}}+Z_{1-\beta}\right)^{2} \times\left(\sigma_{1}^{2}+\sigma_{2}^{2}\right)}{\delta^{2}}$

In this study, a two-tailed test was chosen; $\sigma_{1}$ and $\sigma_{1}$ represent the difference in the change in HAMD-17 scores after treatment in the test group (acupuncture group) and placebo group (control group), respectively, and $\delta$ represents the difference between the two groups. Power Analysis and Sample Size software (PASS; version 11; NCSS Statistical Software, Kaysville, UT, USA) was used for sample size calculation. According to the results of a pilot study [24], $\sigma_{1}=9.50, \sigma_{2}=7.42, \delta=2.08, a=0.05, Z_{1-\frac{a}{2}}=1.96$, $\beta=0.1$, and $Z_{1-\beta}=1.28$. The calculated sample size of each group was approximately 22 individuals. After accounting for an anticipated dropout rate of $20 \%$, the total number of patients required for this trial was 56 , with 28 in each group.

\section{Recruitment}

The researchers will distribute trial information leaflets to the inpatient and outpatient clinics of the hospital. Posters will also be used of recruitment. Suitable participants will be encouraged to ask question about the study and given time to decide if they wish to participate. Participants who are willing to participate will be asked to provide written consent.

\section{Allocation}

\section{Sequence generation}

Participants will be randomly allocated to either the acupuncture group or the control group in a 1:1 ratio by simple randomization. The randomization schedule was calculated in the statistical software SPSS 
(IBM SPSS statistics version 22.0, USA) by a statistical expert from the Faculty of Medical Statistics and Epidemiology, The Second Affiliated Hospital of Guangzhou University of Chinese Medicine.

\section{Allocation concealment mechanism and implementation}

The allocation sequences are enclosed in sealed envelopes with a unique identification number. The envelope will be sent to an investigator in charge of the group assignments. The investigator will specify in the envelope who and when to open the randomly assigned envelope. The number will appear on all report forms to maintain participant confidentiality.

\section{Blinding}

The acupuncturists are not blinded to group allocation. Participants are blinded to their group allocation but are informed that they had an equal chance of allocation to the acupuncture or control group before study participation. The outcome assessor will also be blinded to group allocation. Adverse reactions are carefully observed, and emergency unblinding is required if serious adverse reactions occur. Unblinding will be performed at the end of the trial to perform statistical analysis.

\section{Data collection methods}

Before study initiation, all researchers and acupuncturists will participate in a 2-day training session including assessment and collection of outcome, baseline, and follow-up organized by the Secondary Medical College of Guangzhou University of Traditional Chinese Medicine. During the follow-up period, participants are requested to return to the clinic to receive assessment and will be given subsidies to cover transportation. If the participants could not complete the entire study, and lost to follow-up, these data will be included in the statistics and should be analyzed intentionally.

\section{Data management}

The data and safety monitoring committee is composed of independent clinicians and biostatisticians with rich experience in clinical trials. The ethics committee of the Secondary Medical College of Guangzhou University of Traditional Chinese Medicine will perform random audits to ensure compliance with relevant regulations and guidelines. The case report form (CRF) is a paper version and should be properly recorded by the investigator. One researcher will be responsible for collecting data at each stage of the study, while another researcher will input the data into the SPSS software. This process will be carried out under the supervision of project monitors. According to the terms of informed consent, unless the subject's consent is obtained, all the subject's personal information is confidential and will not be disclosed to the public. The sponsor should keep CRF for 5 years after the end of the research.

\section{Statistical analysis}

Descriptive statistics will be used to describe demographic data of sample groups, while the normality of the distribution of each parameter will be checked by histogram and the Kolmogorov-Smirnov test. A chisquared test will be used to assess sample characteristics and gender. The Mann-Whitney U test will be used to test between-group differences for the HAMD-17 scores and the CC, TE, PE, and NRPE from the WCST. The latency and amplitude of the ERP data will be analyzed by mixed-effect model analysis. $P<$ 0.05 will be taken as the standard for a significant difference. Data analysis will follow the intention-to- 
treat principle including participants who are randomly assigned. Missing data will be input with the last observation carried forward method.

\section{Ethics and dissemination}

This study was approved by the ethics committee of the Secondary Medical College of Guangzhou University of Traditional Chinese Medicine, China, on 6 September 2019 (approval No. ZF2019-170-01). Protocol revision, adverse reaction reports and annual review will be supervised by the ethics committee of the Secondary Medical College of Guangzhou University of Traditional Chinese Medicine. The research observers will explain the study to participants before they voluntarily sign the informed consent form at the beginning of the study. Participation in the study is voluntary and can be discontinued at any time. The decision not to participate will not affect patient's care. On the consent form, participants will be asked to grant permission for the research team to share relevant data with the researchers taking part in the research or regulatory authorities, where relevant. Participants will also be asked if they agree to the use of their data, should they choose to withdraw from the trial. This trial does not involve the collection of biological specimens for storage. Information provided by participants will only be shared with members of the research team. We will make every effort to keep the participants' information confidential. All paper documents related to the study will be kept in a locked cabinet. All participants' information will be kept strictly confidential.

\section{Discussion}

PSD is the most common emotional complication after stroke and characterized by sustained depression and loss of interest. It ranks first among the comorbidities of neurological diseases and depression and is related to poor functional outcomes and high recurrence and mortality. Antidepressants are commonly used in the treatment of depression, which can cause AEs and substance abuse. Therefore, it is very important to find other effective and safe treatments and explore the mechanism of antidepressants.

Acupuncture is one of the most popular nonpharmaceutical therapies for depression and has been increasingly recognized globally in recent years [25]. The literature shows that acupuncture has a positive effect on and improves depressive symptoms of PSD [11]. A recent meta-analysis demonstrated that acupuncture resulted in better outcomes compared with several groups of antidepressant medications without any obvious adverse reactions [13]. However, these trials did not consider placebo or sham acupuncture as control conditions to exclude the psychological effects of acupuncture.

Emotional and cognitive disorders are common psychological features underlying the depressive symptoms of MDD. ERP studies have shown that depressed patients have a tendency to overprocess negative stimulation, and the recognition accuracy of negative and positive information is decreased [26]. It has been found that those with MDD have difficulty shifting attention from negative information, which leads to persistent negative emotional rumination. We believe that this emotional cognitive processing deficit in PSD may manifest as an attentional bias to negative emotions. The existing studies on PSD are 
mainly based on responses to clinical scales to assess depressive symptoms but have not focused on the effects of acupuncture on cognition in those with PSD. However, the mechanisms of how acupuncture regulates attentional bias in those with PSD is still not clear. Therefore, this trial was designed as a randomized, placebo-controlled clinical trial to explore the clinical efficacy and safety of acupuncture therapy on PSD. Moreover, we will employ indices reflective of cognitive function as secondary outcomes, including the WCST and ERP measures, with the expectation that they will provide reliable clinical evidence for acupuncture treatment that helps improve PSD.

There are also some limitations to the study. First, it is difficult to implement the double-blind approach as the acupuncturists could not be blinded, which may in performance bias. Moreover, due to the limitations of project funding and trial period, the follow-up period is only 1 month. Future trials should investigate the benefits of acupuncture treatment for PSD beyond 3 months.

\section{Trial Status}

Trial registration: This study was registered with the Chinese Clinical Trial Registry on 27 October 2019 with the ID ChiCTR1900026948. The protocol version is 1.0, 1 July 2019. Patient recruitment was initiated on 1 December 2019 and is scheduled to be completed by the end of December 2021. If we should amend the protocol, we will communicate with the investigators, ethics committee, and trial registries.

\section{Abbreviations}

PSD: poststroke depression; WCST: Wisconsin Card Sorting Test; HAMD: Hamilton Depression Scale; MDD: major depression disorder; ERP: event-related potential; CT: computed tomography; MRI: magnetic resonance imaging; $\mathrm{CC}$ : categories completed; TE: total errors; PE: perseverative errors; NRPE: nonperseverative errors; RT: reaction times.

\section{Declarations}

\section{Ethics approval and consent to participate}

The study protocol was approved by the ethics committee of the Secondary Medical College of Guangzhou University of Traditional Chinese Medicine, China, on 6 September 2019 (approval No. ZF2019-170-01). Written informed consent will be obtained from all participants.

\section{Consent for publication}

Consent for publication information is included in the written informed consent process. However, no data is being published at this time.

\section{Availability of data and materials}


According to the terms of informed consent, unless the subject's consent is obtained, all the subject's personal information is confidential and will not be disclosed to the public. When necessary, the ethics committee or project funding department may consult the subject's data. They will not use the subject's information for other purposes or disclose it to other groups without permission.

\section{Competing interests}

All the authors of the article declare that they have no competing interests.

\section{Funding}

This research project was supported by the National Natural Science Foundation of China (Grant No.: 82004466), China Postdoctoral Science Foundation Funded Project (Grant No.:

2019M652867), the Foundation for Basic and Applied Basic Research of Guangdong Province (Grant No.: 2019A1515110366), and the Scientific Research Project of Guangdong Traditional Chinese Medicine Bureau (Grant No.: 20201118). The funding bodies did not play a role in the design of the study and collection, analysis, and interpretation of data and in writing the manuscript.

\section{Authors' contributions}

All authors read and approved the final manuscript. All authors meet the four primary ICMJE criteria for authorship.

In addition, all authors have been and are actively involved in the study in different capacities: LC drafted the paper with contributions and approval from all authors. YL and LW were involved in the development of the study material and evaluation protocols and the data collection. LW is responsible for data mining. WF is the lead statistician. LC and WF managed the trial.

\section{Acknowledgments}

We would like to thank all the clinical staff and participants for their contribution to the study.

\section{References}

1. Vorkas PA, Shalhoub J, Lewis MR, Spagou K, Want EJ, Nicholson JK, et al. Metabolic Phenotypes of Carotid Atherosclerotic Plaques Relate to Stroke Risk: An Exploratory Study. EUR J VASC ENDOVASC. 2016.

2. Villa RF, Ferrari F, Moretti A. Post-stroke depression: Mechanisms and pharmacological treatment. Pharmacol Ther. 2018;184:131-44.

3. Ayerbe L, Ayis S, Wolfe CD, Rudd AG. Natural history, predictors and outcomes of depression after stroke: systematic review and meta-analysis. The British journal of psychiatry. 2013;202:14-21.

4. Towfighi A, Ovbiagele B, Husseini NE, Hackett ML, Jorge RE, Kissela BM, et al. Poststroke Depression: A Scientific Statement for Healthcare Professionals From the American Heart Association/American 
Stroke Association. STROKE. 2017;2:e30-43.

5. Yang S, Hua P, Shang X, Cui Z, Zhong S, Gong G, et al. A significant risk factor for poststroke depression: the depression-related subnetwork. J Psychiatry Neurosci. 2015;40:259-68.

6. Bartoli F, Lillia N, Lax A, Crocamo C. Depression after Stroke and Risk of Mortality: A Systematic Review and Meta-Analysis. Stroke Research and Treatment. 2013;2013.

7. Engelter ST, Gostynski M, Papa S, Frei M, Born C, Ajdacic-Gross V, et al. Epidemiology of aphasia attributable to first ischemic stroke: incidence, severity, fluency, etiology, and thrombolysis. STROKE. 2006;37:1379-84.

8. Nys GM, van Zandvoort MJ, de Kort PL, Jansen BP, de Haan EH, Kappelle LJ. Cognitive disorders in acute stroke: prevalence and clinical determinants. CEREBROVASC DIS. 2007;23:408-16.

9. Su TP, Chen MH, Li CT, Lin WC, Hong CJ, Gueorguieva R, et al. Correction: Dose-Related Effects of Adjunctive Ketamine in Taiwanese Patients with Treatment-Resistant Depression.

NEUROPSYCHOPHARMACOL. 2018.

10. MacQueen G, Santaguida P, Keshavarz H, Jaworska N, Levine M, Beyene J, et al. Systematic Review of Clinical Practice Guidelines for Failed Antidepressant Treatment Response in Major Depressive Disorder, Dysthymia, and Subthreshold Depression in Adults. Can J Psychiatry. 2017;62:11-23.

11. Chan YY, Lo WY, Yang SN, Chen YH, Lin JG. The benefit of combined acupuncture and antidepressant medication for depression: A systematic review and meta-analysis. J Affect Disord. 2015;176:106-17.

12. Wang X, Wang Z, Liu J, Chen J, Liu X, Nie G, et al. Repeated acupuncture treatments modulate amygdala resting state functional connectivity of depressive patients. Neuroimage Clin. 2016;12:746-52.

13. Zhang XY, Li YX, Liu DL, Zhang BY, Chen DM. The effectiveness of acupuncture therapy in patients with post-stroke depression: An updated meta-analysis of randomized controlled trials. Med (Baltim). 2019;98:e15894.

14. Shi Y, Zeng Y, Wu L, Liu Z, Zhang S, Yang J, et al. A Study of the Brain Functional Network of PostStroke Depression in Three Different Lesion Locations. Sci Rep. 2017;7:14795.

15. Disner SG, Beevers CG, Haigh EA, Beck AT. Neural mechanisms of the cognitive model of depression. NAT REV NEUROSCI. 2011;12:467-77.

16. Zhang D, He Z, Chen Y, Wei Z. Deficits of unconscious emotional processing in patients with major depression: An ERP study. J Affect Disord. 2016;199:13-20.

17. Chen J, Ma W, Zhang Y, Wu X, Wei D, Liu G, et al. Distinct facial processing related negative cognitive bias in first-episode and recurrent major depression: evidence from the N170 ERP component. PLOS ONE. 2014;9:e109176.

18. A-W C, JM T, DG A, PC AL. G, K K, et al. SPIRIT 2013 Statement: defining standard protocol items for clinical trials. ANN INTERN MED. 2013;158:200-7. 
19. Guo W, Cao X, Sheng L, Li J, Zhang L, Ma Y. Expert Consensus of diagnosis and treatment of major depression with integrated TCM and Western medicine. Chinese Journal of Integrated Chinese Western Medicine. 2020;02:141-8.

20. Folstein MF, Folstein SE, McHugh PR. "Mini-mental state". A practical method for grading the cognitive state of patients for the clinician. J PSYCHIATR RES. 1975;12:189-98.

21. Hamilton M. Development of a rating scale for primary depressive illness. Br J Soc Clin Psychol. 1967;6:278-96.

22. Kataoka Y, Shimada T, Koide Y, Okubo H, Uehara T, Shioiri T, et al. Differences in executive function among patients with schizophrenia, their unaffected first-degree relatives and healthy participants. Int J Neuropsychopharmacol. 2020.

23. Hu B, Rao J, Li X, Cao T, Li J, Majoe D, et al. Emotion Regulating Attentional Control Abnormalities In Major Depressive Disorder: An Event-Related Potential Study. Sci Rep. 2017;7:13530.

24. Yang L. Clinical study on the treatment of depression after infarction by Shugantiaoshen acupuncture. Guangzhou: Guangzhou University of traditional Chinese Medicine; 2012.

25. Samuels N, Gropp C, Singer SR, Oberbaum M. Acupuncture for psychiatric illness: a literature review. BEHAV MED. 2008;34:55-64.

26. Bourke C, Douglas K, Porter R. Processing of facial emotion expression in major depression: a review. Aust N Z J Psychiatry. 2010;8:681-96.

\section{Figures}




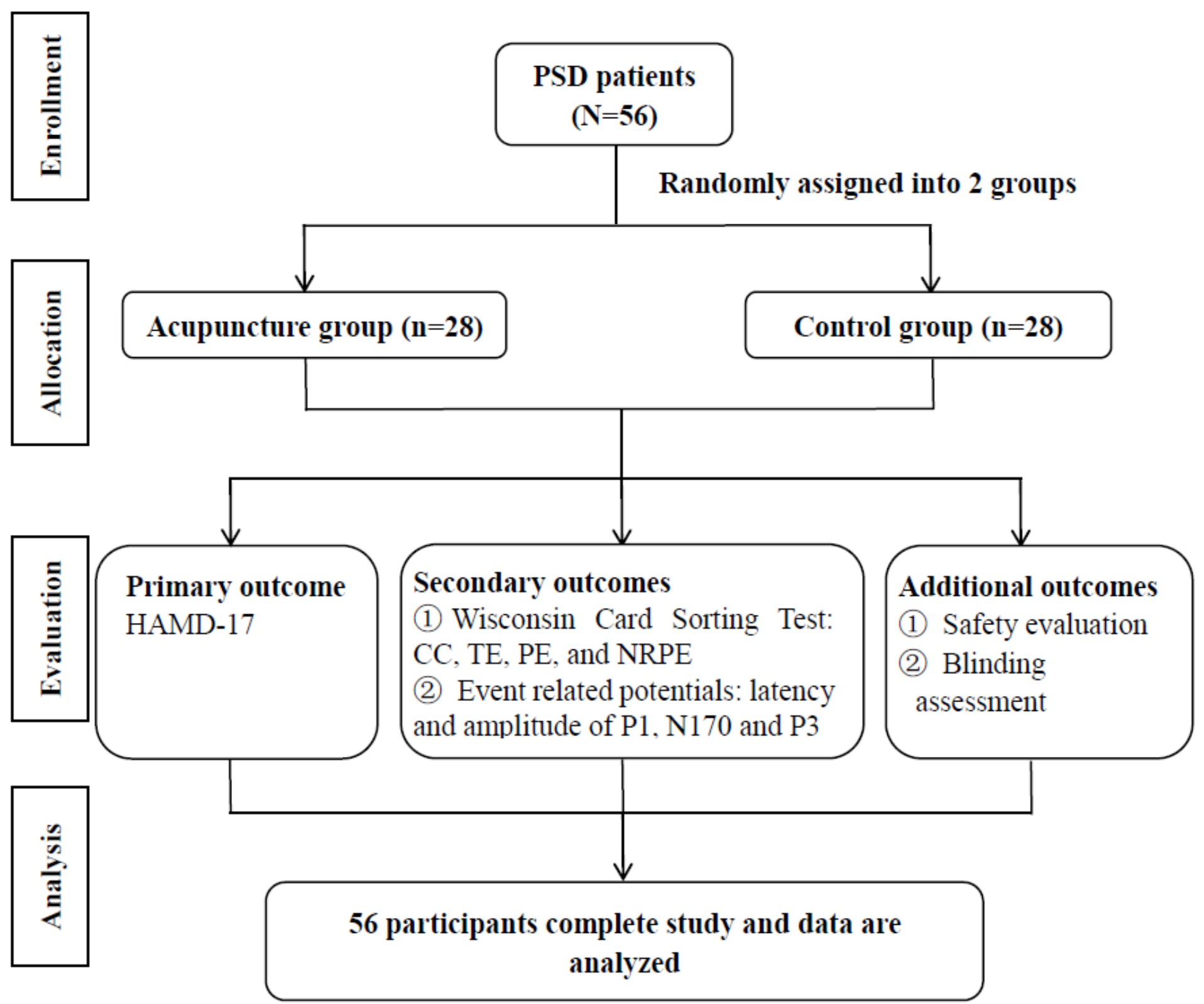

Figure 1

Flow diagram of the study procedure. CC: categories completed, TE: total errors, PE: perseverative errors, NRPE: nonperseverative errors 


\begin{tabular}{|c|c|c|c|c|c|c|c|}
\hline \multirow[b]{3}{*}{ TIMEPOINT (week) } & \multicolumn{7}{|c|}{ STUDY PERIOD } \\
\hline & \multirow{2}{*}{$\frac{\text { Enrolment }}{0}$} & \multirow{2}{*}{$\frac{\text { Allocation }}{0}$} & \multicolumn{4}{|c|}{ Post-allocation } & \multirow{2}{*}{$\frac{\text { Close-out }}{8}$} \\
\hline & & & 1 & 2 & 3 & 4 & \\
\hline \multicolumn{8}{|l|}{ ENROLMENT: } \\
\hline Eligibility screen & $X$ & & & & & & \\
\hline Informed consent & $X$ & & & & & & \\
\hline Allocation & & X & & & & & \\
\hline \multicolumn{8}{|l|}{ INTERVENTIONS: } \\
\hline \multicolumn{8}{|l|}{ Acupuncture group } \\
\hline \multicolumn{8}{|l|}{ Control group } \\
\hline \multicolumn{8}{|l|}{ ASSESSMENTS: } \\
\hline HAMD-17 & & X & & & & $X$ & $X$ \\
\hline WCST & & $X$ & & & & $X$ & $\mathrm{X}$ \\
\hline$E R P$ & & $X$ & & & & $X$ & \\
\hline Adverse events & & & $X$ & $X$ & $X$ & $X$ & \\
\hline Blinding assessment & & & & & & $X$ & \\
\hline
\end{tabular}

Figure 2

Standard Protocol Items: Recommendations for Interventional Trials (SPIRIT) schedule of enrolment, interventions, and assessments. HAMD: Hamilton Depression Scale; WCST: Wisconsin Card Sorting Test; ERP: Event-related potential.

Supplementary Files 
This is a list of supplementary files associated with this preprint. Click to download.

- SPIRITChecklist9.10.doc 